\title{
AFM studies on liquid superlubricity between silica surfaces achieved with surfactant micelles
}

Jinjin Li*, Chenhui Zhang, Peng Cheng, Xinchun Chen, Weiqi Wang, Jianbin Luo*

State Key Laboratory of Tribology, Tsinghua University, Beijing, 100084, China

\section{Corresponding authors:}

*To whom all correspondence should be addressed.

Jinjin Li

Telephone: 8610-62771438, E-mail: lijinjin@mail.tsinghua.edu.cn

Jianbin Luo

Telephone: 8610-62781385, -mail: luojb@tsinghua.edu.cn 
1. SEM image of silica particle attached to the cantilever end

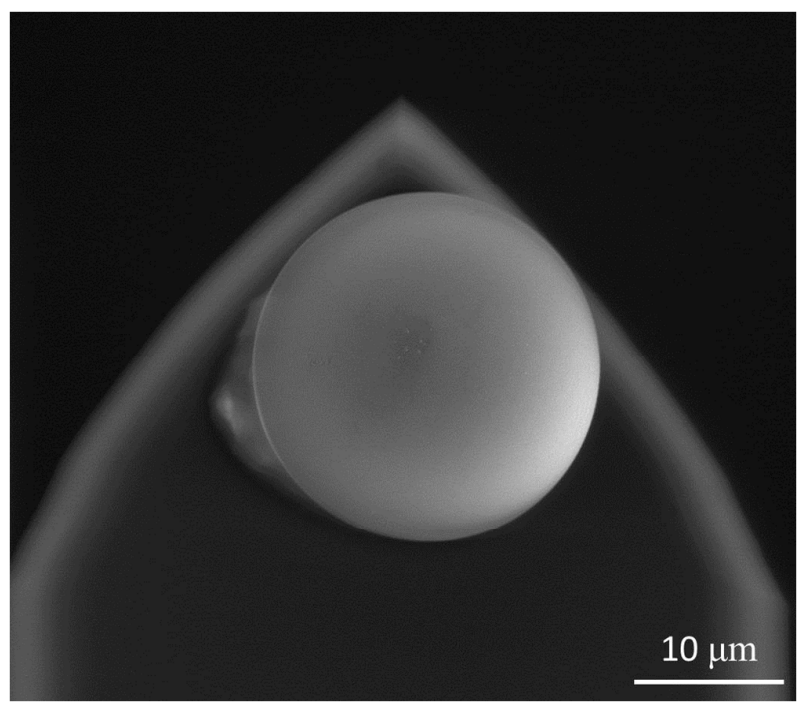

Figure S1. SEM image of silica particle with a diameter of $23 \mu \mathrm{m}$ attached to the cantilever

2. SEM image of special wedge for lateral force calibration

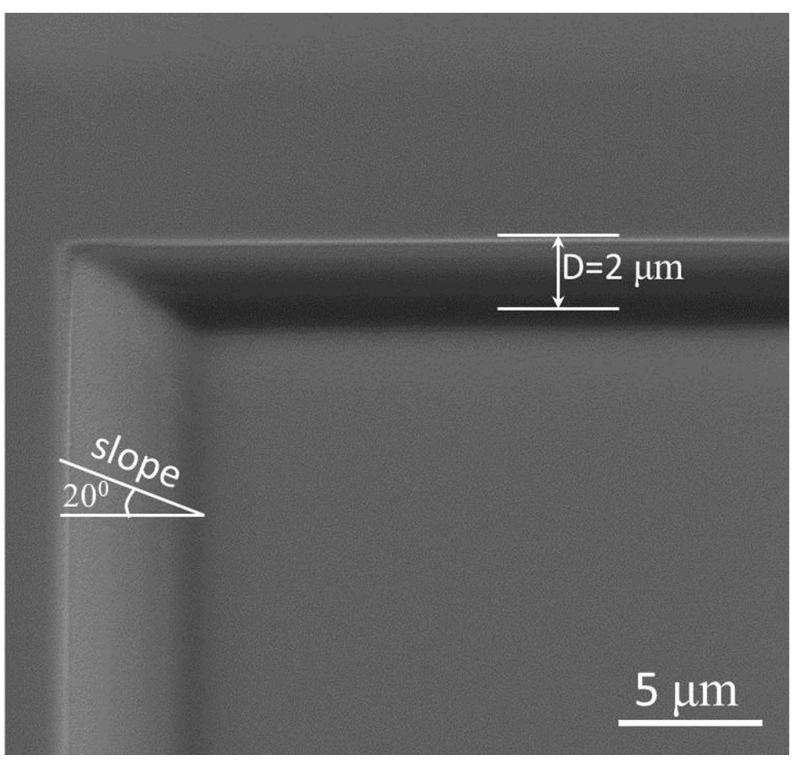

Figure S2. SEM image of special wedge for lateral force calibration. The height of the wedge is 2 $\mu \mathrm{m}$, the incline angle of the slope is $20^{\circ}$, and the size of the etching region is $60 \mu \mathrm{m} \times 30 \mu \mathrm{m}$. The wedge is fabricated by the focused-ion-beam method.

3. Representative friction loop in the super-low friction and high friction region 

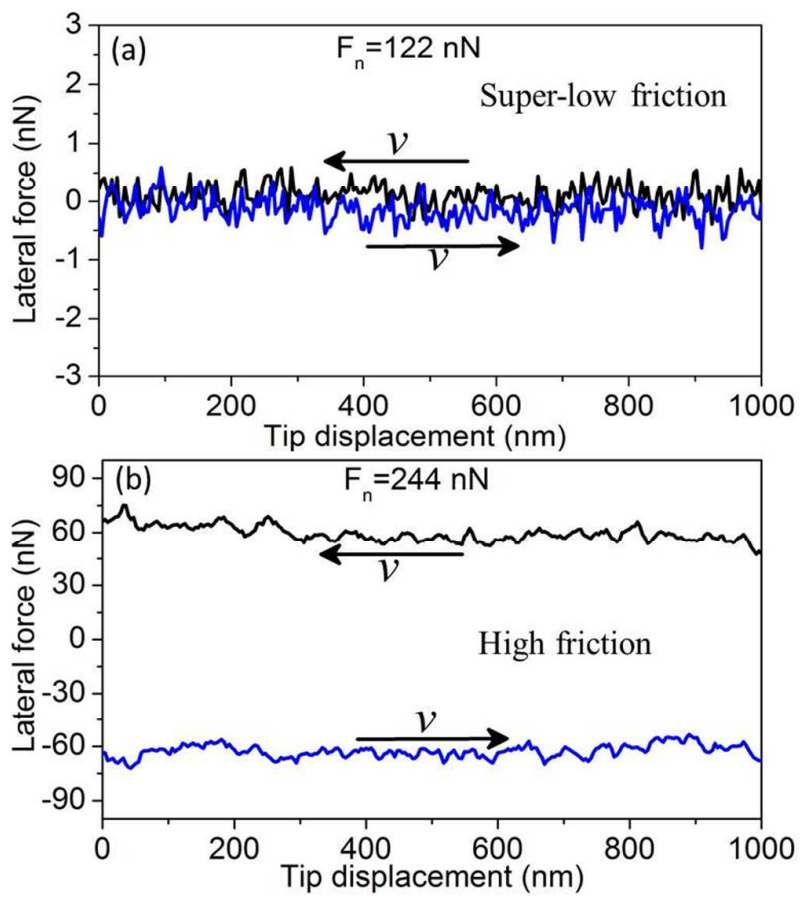

Figure S3. Representative friction loop when the applied load is $122 \mathrm{nN}$ (in the super-low friction region) and $244 \mathrm{nN}$ (in the high friction region), respectively.

4. Effect of hydrodynamic drag on the lateral force

The hydrodynamic drag force $\left(\mathrm{F}_{\mathrm{h}}\right)$ in shear direction can be described by Equation (1).

$$
\mathrm{F}_{\mathrm{h}}=6 \pi \eta v \mathrm{R}
$$

where $\eta$ is the viscosity ( $\eta \approx 1 \mathrm{mPas}$ ), $\mathrm{v}$ is the sliding speed, and $\mathrm{R}$ is the radius of colloidal probe $(\mathrm{R}=11.5 \mu \mathrm{m})$. Considering the maximal speed $\left(\mathrm{v}_{\max }=100 \mu \mathrm{m} / \mathrm{s}\right)$ in Figure $3(\mathrm{~b})$, we can get the maximal hydrodynamic force $\left(\mathrm{F}_{\mathrm{hmax}}=0.02 \mathrm{nN}\right)$. Compared with the measured lateral force (fluctuated within the range of $0.02-0.09 \mathrm{nN}$ ) shown in Figure 3(b), we can found that the maximal hydrodynamic force and the lateral forces are in the same level (noise level). Figure S4 shows the lateral forces with hydrodynamic drag and without hydrodynamic drag under different speeds. It indicates that the influence of hydrodynamic drag force in shear direction is so little that it can be ignored in our work. 


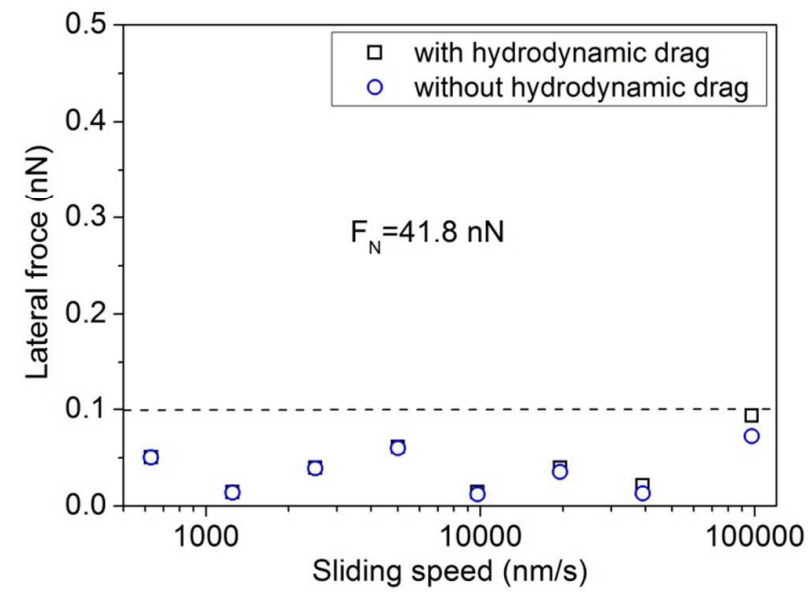

Figure S4. Lateral forces with hydrodynamic drag and without hydrodynamic drag under different speeds when the normal load is set as $41.8 \mathrm{nN}$

5. Lubrication model of $\mathrm{C}_{16} \mathrm{TAB}$ solution $(<1 \mathrm{CMC})$ between two silica surfaces

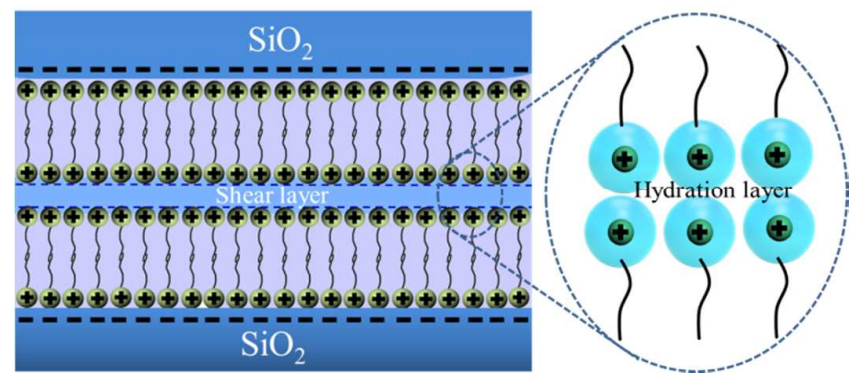

Figure S5. Lubrication model of $\mathrm{C}_{16} \mathrm{TAB}$ solution $(<1 \mathrm{CMC})$ between two silica surfaces when the applied load is less than the critical load (in the super-low friction region). The right part is the illustration of the shear layer between two bilayers.

6. Friction behavior under the lubrication of $\mathrm{C}_{14} \mathrm{TAB}$ and $\mathrm{C}_{12} \mathrm{TAB}$ solution

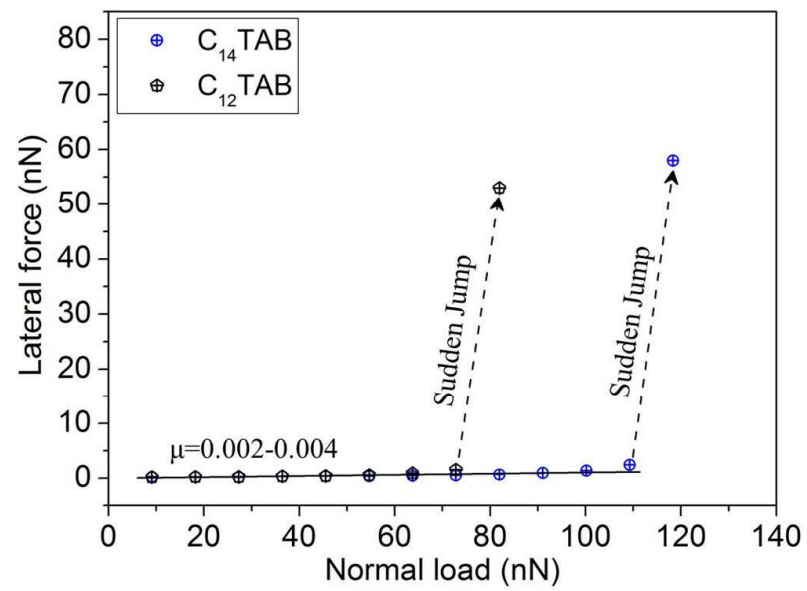

Figure S6. Lateral force as a function of normal load between two silica surfaces immersed in $\mathrm{C}_{14} \mathrm{TAB}$ and $\mathrm{C}_{12} \mathrm{TAB}$ solution (3 CMC) 